\title{
Financial stress among skin cancer patients: a cross-sectional review of the 2013-2018 National Health Interview Survey
}

\author{
Thomas B. Cwalina ${ }^{1,2}\left(\right.$ D Tarun K. Jella ${ }^{1,2} \cdot$ Raghav Tripathi $^{1,2,3} \cdot$ Bryan T. Carroll $^{1,2}$
}

Received: 17 February 2021 / Revised: 20 January 2022 / Accepted: 7 February 2022 / Published online: 22 February 2022

( ) The Author(s), under exclusive licence to Springer-Verlag GmbH Germany, part of Springer Nature 2022

\begin{abstract}
Financial stress among skin cancer patients may limit treatment efficacy by forcing the postponement of care or decreasing adherence to dermatologist recommendations. Limited information is available quantifying the anxiety experienced by skin cancer patients from both healthcare and non-healthcare factors. Therefore, the present study sought to perform a retrospective cross-sectional review of the 2013-2018 cycles of the National Health Interview Survey (NHIS) to determine the prevalence, at-risk groups, and predictive factors of skin cancer patient financial stress. Survey responses estimated that $11.45 \%(95 \% \mathrm{Cl}$ $10.02-12.88 \%$ ) of skin cancer patients experience problems paying medical bills, $20.34 \%$ (95\% Cl 18.97-21.71\%) of patients worry about the medical costs, $13.73 \%(95 \% \mathrm{Cl} 12.55-14.91 \%)$ of patients worry about housing costs, and $37.48 \%(95 \% \mathrm{Cl}$ $35.83-39.14 \%$ ) of patients worry about money for retirement. Focusing on at-risk groups, black patients, uninsured patients, and patients with low incomes $(<200 \%$ poverty level) consistently experienced high rates of financial stress for each of the four measures. Multivariable logistic regression revealed low education, lack of insurance, and low income to be predictive of financial stress. These findings suggest that a considerable proportion of skin cancer patients experience financial stress related to both healthcare and non-healthcare factors. Where possible, the additional intricacy of treating patients at risk of high financial stress may be considered to optimize patient experience and outcomes.
\end{abstract}

Keywords Skin cancer $\cdot$ Oncology $\cdot$ Financial stress $\cdot$ National Health Interview Survey $\cdot$ Health equity $\cdot$ Social determinants

\section{Introduction}

The impact of financial stress within the field of dermatology and dermatologic oncology is of particular importance due to the increasing prevalence of skin cancer and the higher amount of out-of-pocket costs these patients may experience [1-4]. A study analyzing over 9 million new cancer diagnoses between 2000 and 2012 estimated that in the first two years following their diagnosis, $42.4 \%$ of patients reported depleting their life savings [5]. These high expenditures can often increase

Thomas B. Cwalina

thomas.b.cwalina@gmail.com

1 Case Western Reserve University School of Medicine, Cleveland, OH, USA

2 Department of Dermatology, University Hospitals Case Medical Center, 11100 Euclid Ave, Cleveland, OH 44106, USA

3 The University of Iowa Hospitals and Clinics, Iowa City, IA, USA patients' worries and anxieties, which can further worsen their wellbeing and exacerbate disparities [1]. Since financial stress can limit the effectiveness of clinical treatment greatly, increasing efforts have been made to screen for financial stress factors and align treatment plans to a patient's situation $[6,7]$.

Previous research has analyzed the troubling impacts of low socioeconomic status on skin cancer outcome disparities [8-10]. However, few prior investigations have been able to quantify the potential financial stress reported directly from skin cancer patients, from both healthcare and nonhealthcare factors. It is hypothesized that within the skin cancer population a considerable portion of patients experiences financial stress, with factors such as health status, income, and insurance serving as predictors. Therefore, the present study sought to perform a cross-sectional review of the National Health Interview Survey (NHIS) to quantify the prevalence of various financial stress measures among skin cancer patients, identify subgroups affected most by personal financial stress, and isolate the most predictive features of skin cancer patients experiencing financial stress. 


\section{Methods}

\section{Study design}

This study was conducted across the 2013 to 2018 cycles of the National Health Interview Survey (NHIS), a nationally representative survey conducted annually by the National Center for Health Statistics (NCHS) [11]. Using complex sampling design techniques such as stratification and clustering, the NHIS collects estimates of a wide range of health information for the civilian noninstitutionalized population of the United States. With over 600 unique questions included, the NHIS has been employed to analyze health concerns from a patient's perspective [12-15]. Since, 2013 the NHIS included financial stress questions about the following topics: "problems paying or unable to pay medical bills, past 12 months"; "worried about the medical costs of health care"; "worried about paying rent/mortgage/housing costs"; "worried about money for retirement". For the first question about "problems paying or unable to pay medical bills, past 12 months", responses were structured in a yes/ no format. For the remaining three questions about financial worries, responses were structured in a four-step scale indicating the amount of worry: "not worried at all", "not too worried", "moderately worried", or "very worried".

These respondents from the NHIS were then filtered to only include those who reported having ever been told by a doctor or other health care professional that they had a form of skin cancer (melanoma or non-melanoma) [16]. Within this subset of patients, only those who completed a response to the aforementioned financial security questions were included. Finally, the responses to the four-step scale questions about financial worries were then binarized into "not worried at all"/"not too worried" and "moderately worried"/"very worried" according to previous analysis of the NHIS to increase compatibility for statistical analysis [17].

\section{Statistical analysis}

First, this subset of skin cancer patients was then analyzed to see the prevalence of financial stress concerns among the population. The overall proportions of the responses were calculated for all four financial stress questions, including their $95 \%$ confidence intervals. Next, the data was analyzed for any crude differences in financial stress responses based on the independent variables included in the study. The $95 \%$ confidence intervals were calculated for the breakdowns and statistical significance was determined using a Pearson $X^{2}$ test with the Rao Scott adjustment [18]. To account for potential confounding factors, a multivariable analysis was conducted using logistic regression while controlling for all the independent variables to predict the financial stress responses. From this regression, the adjusted odds ratios with $95 \%$ confidence intervals were calculated and statistical significance was determined using the Wald test [19].

Statistical analysis was conducted using R version 4.0.2. All statistical calculations accounted for the complex sampling design of the NHIS using the R "survey" package (version 4.0). Finally, statistical tests were two-sided, and significance was defined as $p<0.05$.

\section{Results}

The unconditional response rate of the survey ranged from 64.2 to $75.7 \%$ over the study period. The cohort of skin cancer patients who responded to the four survey questionsproblems paying medical bills, worried about the medical costs, worried about housing costs, and worried about money for retirement—included a total of 5698, 5601, 5605, 5604 patients, respectively. The average age for all question cohorts was 65 years old. $96 \%$ of respondents in all cohorts were non-Hispanic white. In addition, $48 \%$ of respondents were female in all cohorts (Table 1).

From those skin cancer patients sampled, $11.45 \%$ (95\% Cl 10.02-12.88\%) of respondents reported problems paying medical bills, 20.34\% (95\% Cl 18.97-21.71\%) of respondents reported being worried about the medical costs, $13.73 \%$ (95\% $\mathrm{Cl} 12.55-14.91 \%$ ) of respondents reported being worried about housing costs, and $37.48 \%(95 \% \mathrm{Cl}$ $35.83-39.14 \%$ ) of respondents reported being worried about money for retirement (Table 2). Focusing on patient subgroups, black patients, uninsured patients, and patients with income $<200 \%$ poverty level experienced high rates of financial stress for each of the four measures. For example, $39.20 \%$ (12.10-66.30\%) of black patients indicated having problems paying medical bills in the past 12 months. In addition, $33.84 \%$ (30.62-37.06\%) of patients with income below $200 \%$ of the poverty level responded being worried about medical costs. Finally, 51.41\% (40.55-62.23\%) of uninsured patients expressed worry about housing costs and $73.22 \%$ (64.85-82.60\%) conveyed worry about money for retirement costs (Table 2).

After controlling for potential confounding effects of the variables in the logistic regression, educational attainment, insurance status, income level, and self-reported health status were statistically predictive for each measure of financial stress. Demonstrating their effects on financial stress, patients without a high school diploma had increased odds [aOR $2.55(1.43-4.57), p<0.001$ ] of having problems paying or being unable to pay medical bills in the past 12 months than patients with a graduate degree. Uninsured patients had higher odds [aOR 4.82 
Table 1 Cohort demographics of skin cancer patients from 2013 to 2018 responding to financial stress survey questions

\begin{tabular}{|c|c|c|c|c|}
\hline Weighted \% (95\% CI) & $\begin{array}{l}\text { Problems paying medical } \\
\text { bills }\end{array}$ & $\begin{array}{l}\text { Worried about healthcare } \\
\text { costs }\end{array}$ & $\begin{array}{l}\text { Worried about housing } \\
\text { costs }\end{array}$ & $\begin{array}{l}\text { Worried about retirement } \\
\text { costs }\end{array}$ \\
\hline Sample size & 5698 & 5601 & 5605 & 5604 \\
\hline Age (mean, 95\% CI) & $65.05(64.60-65.50)$ & $65.08(64.62-65.54)$ & $65.09(64.63-65.55)$ & $65.08(64.63-65.54)$ \\
\hline \multicolumn{5}{|l|}{ Race/ethnicity } \\
\hline White & $95.72(95.04-96.41)$ & 95.67 (94.98-96.37) & 95.68 (94.98-96.37) & 95.68 (94.98-96.37) \\
\hline Black & $0.41(0.18-0.63)$ & $0.41(0.18-0.64)$ & $0.41(0.18-0.64)$ & $0.41(0.18-0.64)$ \\
\hline Hispanic & $1.97(1.53-2.41)$ & $1.99(1.54-2.44)$ & $1.99(1.54-2.43)$ & $1.99(1.54-2.44)$ \\
\hline Other & $1.90(1.41-2.39)$ & $1.93(1.43-2.42)$ & $1.93(1.43-2.42)$ & $1.93(1.43-2.42)$ \\
\hline \multicolumn{5}{|l|}{ Sex } \\
\hline Male & $51.99(50.23-53.75)$ & $52.18(50.41-53.96)$ & $52.21(50.43-53.98)$ & $52.21(50.43-53.98)$ \\
\hline Female & $48.01(46.25-49.77)$ & $47.82(46.04-49.59)$ & $47.79(46.02-49.57)$ & $47.79(46.02-49.57)$ \\
\hline \multicolumn{5}{|l|}{ Sexual orientation } \\
\hline Straight & $95.50(94.86-96.15)$ & $96.96(96.42-97.50)$ & 96.98 (96.45-97.52) & $96.96(96.42-97.50)$ \\
\hline $\begin{array}{l}\text { Lesbian, gay, bisexual, or } \\
\text { other }\end{array}$ & $4.50(3.85-5.14)$ & $3.04(2.50-3.58)$ & $3.02(2.48-3.55)$ & $3.04(2.50-3.58)$ \\
\hline \multicolumn{5}{|l|}{ US citizen } \\
\hline Yes & 99.37 (99.12-99.63) & 99.42 (99.18-99.65) & $99.42(99.18-99.65)$ & $99.42(99.18-99.65)$ \\
\hline No & $0.63(0.37-0.88)$ & $0.58(0.35-0.82)$ & $0.58(0.35-0.82)$ & $0.58(0.35-0.82)$ \\
\hline \multicolumn{5}{|l|}{ Born in the US } \\
\hline Yes & $95.38(94.64-96.11)$ & $95.43(94.71-96.16)$ & $95.43(94.71-96.16)$ & $95.43(94.71-96.16)$ \\
\hline No & $4.62(3.89-5.36)$ & $4.57(3.84-5.29)$ & $4.57(3.84-5.29)$ & $4.57(3.84-5.29)$ \\
\hline \multicolumn{5}{|l|}{ Region } \\
\hline Northeast & $15.35(13.99-16.72)$ & $15.30(13.95-16.65)$ & $15.30(13.95-16.65)$ & $15.29(13.94-16.64)$ \\
\hline Midwest & $23.90(22.26-25.55)$ & $23.73(22.10-25.36)$ & $23.74(22.11-25.37)$ & $23.75(22.11-25.38)$ \\
\hline South & $38.68(36.60-40.77)$ & 38.77 (36.69-40.85) & $38.79(36.71-40.88)$ & $38.78(36.69-40.86)$ \\
\hline West & $22.06(20.34-23.79)$ & $22.20(20.46-23.94)$ & $22.17(20.44-23.91)$ & $22.19(20.45-23.93)$ \\
\hline \multicolumn{5}{|l|}{$\%$ Federal poverty level } \\
\hline $200 \%$ or above & $81.20(79.94-82.46)$ & $81.39(80.13-82.66)$ & $81.34(80.08-82.61)$ & $81.39(80.13-82.64)$ \\
\hline$<200 \%$ & $18.80(17.54-20.06)$ & $18.61(17.34-19.87)$ & $18.66(17.39-19.92)$ & $18.61(17.36-19.87)$ \\
\hline \multicolumn{5}{|l|}{ Insurance } \\
\hline Private & $66.03(64.42-67.65)$ & $65.98(64.36-67.60)$ & $65.96(64.34-67.58)$ & $65.99(64.37-67.61)$ \\
\hline Public & $31.56(29.98-33.15)$ & $31.61(30.03-33.20)$ & $31.63(30.05-33.22)$ & $31.59(30.00-33.18)$ \\
\hline Uninsured & $2.40(1.90-2.91)$ & $2.41(1.90-2.92)$ & $2.41(1.89-2.92)$ & $2.42(1.90-2.93)$ \\
\hline \multicolumn{5}{|l|}{ Marital status } \\
\hline Married & $66.55(65.05-68.05)$ & $66.71(65.21-68.20)$ & $66.68(65.19-68.18)$ & $66.68(65.19-68.18)$ \\
\hline Single & $33.45(31.95-34.95)$ & $33.29(31.80-34.79)$ & $33.32(31.82-34.81)$ & $33.32(31.82-34.81)$ \\
\hline \multicolumn{5}{|l|}{ Self-reported health status } \\
\hline Good to excellent & $83.56(82.33-84.79)$ & $83.59(82.34-84.84)$ & $83.56(82.32-84.81)$ & $83.57(82.33-84.82)$ \\
\hline Poor to fair & $16.44(15.21-17.67)$ & $16.41(15.16-17.66)$ & $16.44(15.19-17.68)$ & $16.43(15.18-17.67)$ \\
\hline \multicolumn{5}{|c|}{ Highest educational attainment } \\
\hline Graduate degree & $18.95(17.55-20.34)$ & $19.07(17.66-20.48)$ & $19.06(17.65-20.47)$ & $19.06(17.65-20.47)$ \\
\hline Bachelor's degree & $22.45(21.06-23.85)$ & $22.54(21.14-23.94)$ & $22.53(21.13-23.93)$ & $22.55(21.14-23.95)$ \\
\hline HS diploma & $50.95(49.11-52.79)$ & $50.89(49.04-52.74)$ & $50.90(49.05-52.75)$ & $50.88(49.04-52.73)$ \\
\hline No HS diploma & $7.65(6.79-8.51)$ & $7.50(6.64-8.37)$ & $7.51(6.64-8.37)$ & $7.51(6.64-8.37)$ \\
\hline
\end{tabular}

(3.02-7.70, $p<0.001]$ of being worried about medical costs than patients with private insurance. In addition, those patients with income $<200 \%$ of the poverty level had increased odds [aOR $2.40(1.88-3.06), p<0.001]$ of being worried about housing costs than patients with income $200 \%$ and above. Finally, patients with fair/poor self-reported health status had increased odds [aOR 1.70 (1.38-2094), $p<0.001]$ of being worried about retirement costs than patients with reported good/excellent health (Table 3). 
Table 2 Prevalence of financial stress in skin cancer patients from 2013 to 2018 by demographic and socioeconomic subgroup

\begin{tabular}{|c|c|c|c|c|}
\hline Weighted \% (95\% CI) & $\begin{array}{l}\text { Problems paying medical } \\
\text { bills }\end{array}$ & $\begin{array}{l}\text { Worried about healthcare } \\
\text { costs }\end{array}$ & $\begin{array}{l}\text { Worried about housing } \\
\text { costs }\end{array}$ & $\begin{array}{l}\text { Worried about retirement } \\
\text { costs }\end{array}$ \\
\hline Sample size & 5698 & 5601 & 5605 & 5604 \\
\hline Overall & $11.45(10.02-12.88)$ & $20.34(18.97-21.71)$ & $13.73(12.55-14.91)$ & $37.48(35.83-39.14)$ \\
\hline \multicolumn{5}{|l|}{ Race/ethnicity } \\
\hline White & $11.01(9.54-12.48)$ & $19.96(18.56-21.35)$ & $13.27(12.07-14.46)$ & $37.24(35.52-38.95)$ \\
\hline Black & $39.20(12.10-66.30)$ & $42.56(12.22-72.89)$ & $35.52(3.62-67.43)$ & $48.67(20.04-77.31)$ \\
\hline Hispanic & $13.82(6.93-20.71)$ & $25.82(15.81-35.83)$ & $22.50(13.22-31.78)$ & $38.56(27.88-49.24)$ \\
\hline Other & $25.39(13.23-37.55)$ & $29.07(17.43-40.72)$ & $22.89(12.19-33.59)$ & $46.21(33.55-58.87)$ \\
\hline \multicolumn{5}{|l|}{ Sex } \\
\hline Male & $10.96(8.58-13.34)$ & $17.66(15.83-19.50)$ & $11.70(10.1-13.3)$ & $33.13(30.83-35.44)$ \\
\hline Female & $11.99(10.46-13.51)$ & $23.27(21.18-25.35)$ & $15.95(14.2-17.7)$ & $42.23(39.76-44.70)$ \\
\hline \multicolumn{5}{|l|}{ Sexual orientation } \\
\hline Straight & $11.08(9.61-12.55)$ & $20.26(18.87-21.64)$ & $13.54(12.35-14.74)$ & $37.05(35.35-38.75)$ \\
\hline $\begin{array}{l}\text { Lesbian, gay, bisexual, or } \\
\text { other }\end{array}$ & $19.39(13.00-25.79)$ & $23.11(15.38-30.83)$ & $19.65(12.10-27.21)$ & $51.25(41.83-60.66)$ \\
\hline \multicolumn{5}{|l|}{ US citizen } \\
\hline Yes & $11.48(10.04-12.93)$ & $20.31(18.93-21.69)$ & $13.74(12.56-14.92)$ & $37.47(35.81-39.13)$ \\
\hline No & $6.69(-2.06-15.44)$ & $25.83(9.24-42.42)$ & $11.82(1.55-22.09)$ & $39.82(20.42-59.21)$ \\
\hline \multicolumn{5}{|l|}{ Born in the US } \\
\hline Yes & $11.56(10.11-13.02)$ & $19.95(18.56-21.35)$ & $13.67(12.46-14.87)$ & 37.37 (35.67-39.07) \\
\hline No & $9.14(4.95-13.34)$ & $28.47(21.39-35.56)$ & $15.03(9.73-20.33)$ & $39.75(31.76-47.75)$ \\
\hline \multicolumn{5}{|l|}{ Region } \\
\hline Northeast & $6.25(4.06-8.45)$ & $19.25(15.26-23.25)$ & $17.65(13.79-21.52)$ & $39.12(34.55-43.68)$ \\
\hline Midwest & $12.54(10.44-14.63)$ & $17.97(15.69-20.25)$ & $13.34(11.10-15.58)$ & $35.99(32.67-39.32)$ \\
\hline South & $13.46(10.41-16.51)$ & $22.41(20.13-24.69)$ & $12.70(10.89-14.50)$ & $36.70(33.99-39.41)$ \\
\hline West & $10.38(8.12-12.64)$ & $20.01(17.03-22.99)$ & $13.24(10.97-15.51)$ & $39.31(36.07-42.55)$ \\
\hline \multicolumn{5}{|l|}{$\%$ Federal poverty level } \\
\hline $200 \%$ or above & $8.40(6.76-10.03)$ & $17.26(15.83-18.69)$ & $10.55(9.30-11.80)$ & $34.34(32.47-36.21)$ \\
\hline$<200 \%$ & $24.66(21.64-27.67)$ & $33.84(30.62-37.06)$ & $27.59(24.55-30.63)$ & $51.23(47.75-54.70)$ \\
\hline \multicolumn{5}{|l|}{ Insurance } \\
\hline Private & $9.61(7.69-11.53)$ & $19.05(17.29-20.82)$ & $11.87(10.43-13.32)$ & $38.70(36.53-40.87)$ \\
\hline Public & $12.94(11.08-14.81)$ & $19.59(17.45-21.74)$ & $14.73(12.82-16.64)$ & $32.20(29.71-34.69)$ \\
\hline Uninsured & $42.56(32.37-52.74)$ & $65.52(54.90-76.13)$ & $51.41(40.55-62.27)$ & $73.22(63.85-82.60)$ \\
\hline \multicolumn{5}{|l|}{ Marital status } \\
\hline Married & $9.64(7.69-11.60)$ & $18.45(16.74-20.17)$ & $11.20(9.73-12.67)$ & $36.21(33.99-38.43)$ \\
\hline Single & $15.05(13.23-16.87)$ & $24.13(21.94-26.32)$ & $18.79(16.73-20.84)$ & $40.02(37.53-42.51)$ \\
\hline \multicolumn{5}{|l|}{ Self-reported health status } \\
\hline Good to excellent & $8.59(6.98-10.19)$ & $17.41(15.97-18.84)$ & $11.76(10.51-13.00)$ & $35.52(33.74-37.30)$ \\
\hline Poor to fair & $26.01(22.68-29.35)$ & $35.28(31.33-39.23)$ & $23.75(20.28-27.23)$ & $47.46(43.41-51.51)$ \\
\hline \multicolumn{5}{|c|}{ Highest educational attainment } \\
\hline Graduate degree & $4.93(3.27-6.59)$ & $11.77(9.26-14.27)$ & $7.30(5.19-9.40)$ & $28.69(25.21-32.17)$ \\
\hline Bachelor's degree & $9.70(7.31-12.08)$ & $17.95(15.01-20.88)$ & $10.36(8.09-12.63)$ & $37.89(34.15-41.63)$ \\
\hline HS diploma & $13.40(11.06-15.74)$ & $23.17(21.25-25.09)$ & $16.72(14.91-18.53)$ & $40.92(38.48-43.35)$ \\
\hline No HS diploma & $19.79(15.36-24.21)$ & $30.16(24.98-35.33)$ & $19.89(15.35-24.43)$ & $35.30(29.80-40.80)$ \\
\hline
\end{tabular}


Table 3 Predictors of various measures of financial stress for skin cancer patients from 2013 to 2018

\begin{tabular}{|c|c|c|c|c|c|c|c|c|}
\hline & \multicolumn{2}{|c|}{$\begin{array}{l}\text { Problems paying medical } \\
\text { bills }\end{array}$} & \multicolumn{2}{|c|}{$\begin{array}{l}\text { Worried about healthcare } \\
\text { costs }\end{array}$} & \multicolumn{2}{|c|}{ Worried about housing costs } & \multicolumn{2}{|c|}{$\begin{array}{l}\text { Worried about retirement } \\
\text { costs }\end{array}$} \\
\hline & Adjusted odds ratio & $p$ value & Adjusted odds ratio & $p$ value & Adjusted odds ratio & $p$ value & Adjusted odds ratio & $p$ value \\
\hline Intercept & $0.25(0.10-0.62)$ & 0.003 & $0.85(0.47-1.52)$ & 0.577 & $1.43(0.73-2.82)$ & 0.300 & $10.10(5.90-17.29)$ & $<0.001$ \\
\hline Age & $0.96(0.95-0.97)$ & $<0.001$ & $0.97(0.96-0.97)$ & $<0.001$ & $0.96(0.95-0.96)$ & $<0.001$ & $0.95(0.94-0.96)$ & $<0.001$ \\
\hline \multicolumn{9}{|l|}{ Race/ethnicity } \\
\hline White & 1 [reference] & NA & 1 [reference] & NA & 1 [reference] & NA & 1 [reference] & NA \\
\hline Black & $2.14(0.70-6.50)$ & 0.181 & $1.42(0.41-4.88)$ & 0.578 & $1.91(0.47-7.79)$ & 0.364 & $1.06(0.39-2.86)$ & 0.906 \\
\hline Hispanic & $0.81(0.38-1.74)$ & 0.584 & $0.66(0.37-1.19)$ & 0.170 & $1.16(0.64-2.13)$ & 0.623 & $0.67(0.39-1.13)$ & 0.135 \\
\hline Other & $1.86(0.87-4.00)$ & 0.111 & $1.11(0.55-2.25)$ & 0.774 & $1.40(0.64-3.03)$ & 0.401 & $1.08(0.59-2.00)$ & 0.794 \\
\hline \multicolumn{9}{|l|}{ Sex } \\
\hline Male & 1 [reference] & NA & 1 [reference] & NA & 1 [reference] & NA & 1 [reference] & NA \\
\hline Female & $0.92(0.68-1.26)$ & 0.614 & $1.23(1.02-1.48)$ & 0.031 & $1.12(0.90-1.40)$ & 0.307 & $1.24(1.05-1.46)$ & 0.013 \\
\hline \multicolumn{9}{|l|}{ Sexual orientation } \\
\hline Straight & 1 [reference] & NA & 1 [reference] & NA & 1 [reference] & NA & 1 [reference] & NA \\
\hline $\begin{array}{l}\text { Lesbian, gay, } \\
\text { bisexual, or } \\
\text { other }\end{array}$ & $1.35(0.83-2.21)$ & 0.232 & $0.76(0.47-1.22)$ & 0.252 & $0.88(0.51-1.51)$ & 0.632 & $1.25(0.82-1.92)$ & 0.298 \\
\hline \multicolumn{9}{|l|}{ US citizen } \\
\hline Yes & 1 [reference] & NA & 1 [reference] & NA & 1 [reference] & NA & 1 [reference] & NA \\
\hline No & $0.61(0.12-3.10)$ & 0.551 & $0.70(0.27-1.82)$ & 0.468 & $0.63(0.20-1.96)$ & 0.422 & $0.85(0.35-2.02)$ & 0.706 \\
\hline \multicolumn{9}{|l|}{ Born in the US } \\
\hline Yes & 1 [reference] & NA & 1 [reference] & NA & 1 [reference] & NA & 1 [reference] & NA \\
\hline No & $0.84(0.43-1.66)$ & 0.619 & $1.84(1.18-2.88)$ & 0.008 & $1.03(0.63-1.69)$ & 0.900 & $1.17(0.76-1.79)$ & 0.485 \\
\hline \multicolumn{9}{|l|}{ Region } \\
\hline Northeast & 1 [reference] & NA & 1 [reference] & NA & 1 [reference] & NA & 1 [reference] & NA \\
\hline Midwest & $1.83(1.21-2.78)$ & 0.004 & $0.80(0.58-1.10)$ & 0.163 & $0.56(0.39-0.80)$ & 0.002 & $0.75(0.59-0.96)$ & 0.025 \\
\hline South & $1.82(1.14-2.90)$ & 0.012 & $1.02(0.76-1.39)$ & 0.873 & $0.49(0.35-0.68)$ & $<0.001$ & $0.79(0.63-0.99)$ & 0.043 \\
\hline West & $1.59(1.01-2.48)$ & 0.044 & $0.98(0.70-1.38)$ & 0.915 & $0.59(0.41-0.84)$ & 0.004 & $0.94(0.74-1.20)$ & 0.634 \\
\hline \multicolumn{9}{|c|}{$\%$ Federal poverty level } \\
\hline $200 \%$ or above & 1 [reference] & NA & 1 [reference] & NA & 1 [reference] & NA & 1 [reference] & NA \\
\hline$<200 \%$ & $2.29(1.67-3.15)$ & $<0.001$ & $1.79(1.45-2.22)$ & $<0.001$ & $2.40(1.88-3.06)$ & $<0.001$ & $2.26(1.85-2.76)$ & $<0.001$ \\
\hline \multicolumn{9}{|l|}{ Insurance } \\
\hline Private & 1 [reference] & NA & 1 [reference] & NA & 1 [reference] & NA & 1 [reference] & NA \\
\hline Public & $1.10(0.84-1.44)$ & 0.481 & $0.91(0.75-1.12)$ & 0.387 & $1.16(0.93-1.46)$ & 0.194 & $0.85(0.72-1.01)$ & 0.063 \\
\hline Uninsured & $3.53(2.18-5.70)$ & $<0.001$ & $4.82(3.02-7.70)$ & $<0.001$ & $4.32(2.71-6.88)$ & $<0.001$ & $2.36(1.42-3.95)$ & 0.001 \\
\hline \multicolumn{9}{|l|}{ Marital status } \\
\hline Married & 1 [reference] & NA & 1 [reference] & NA & 1 [reference] & NA & 1 [reference] & NA \\
\hline Single & $1.13(0.88-1.46)$ & 0.328 & $1.11(0.91-1.34)$ & 0.297 & $1.35(1.07-1.70)$ & 0.011 & $1.03(0.87-1.22)$ & 0.712 \\
\hline \multicolumn{9}{|c|}{ Self-reported health status } \\
\hline Good to excellent & 1 [reference] & NA & 1 [reference] & NA & 1 [reference] & NA & 1 [reference] & NA \\
\hline Poor to fair & $2.87(2.15-3.84)$ & $<0.001$ & $2.29(1.84-2.85)$ & $<0.001$ & $1.84(1.42-2.38)$ & $<0.001$ & $1.70(1.38-2.09)$ & $<0.001$ \\
\hline \multicolumn{9}{|c|}{ Highest educational attainment } \\
\hline Graduate degree & 1 [reference] & NA & 1 [reference] & NA & 1 [reference] & NA & 1 [reference] & NA \\
\hline Bachelor's degree & $1.89(1.19-3.02)$ & 0.008 & $1.57(1.13-2.18)$ & 0.008 & $1.33(0.88-2.01)$ & 0.181 & $1.44(1.13-1.83)$ & 0.003 \\
\hline HS diploma & $2.25(1.40-3.61)$ & $<0.001$ & $2.04(1.54-2.70)$ & $<0.001$ & $2.18(1.53-3.10)$ & $<0.001$ & $1.69(1.35-2.11)$ & $<0.001$ \\
\hline No HS diploma & $2.55(1.43-4.57)$ & 0.002 & $2.36(1.57-3.53)$ & $<0.001$ & $2.03(1.23-3.35)$ & 0.006 & $1.23(0.85-1.78)$ & 0.282 \\
\hline
\end{tabular}




\section{Discussion}

This analysis identified the prevalence, at-risk groups, and potential predictive factors for various measures of healthcare and non-healthcare financial stress. Based on the analysis, a considerable percentage of skin cancer patients experience difficulty paying medical bills and even more worry about medical costs. In addition, numerous skin cancer patients worry about financial costs outside of healthcare, which only adds to the overall anxiety these patients may experience. Disparities also exist within each financial stress response when stratified by subgroups. Those patients who were uninsured were consistently the most affected, followed by black patients and patients with income below $200 \%$ of the federal poverty level. Previous research has demonstrated disparities within these groups in skin cancer outcomes [8]. While skin cancer outcome disparities are complex and multifactorial, the high amount of financial stress within these groups highlights an upstream cause and primary area for improvement.

The results from the multivariable logistic regression provide additional information as to which patients are most likely to be experiencing financial stress, controlling for potential confounding. It was hypothesized, based upon previous research in other fields, that poor health, a lack of insurance, and low education would be most predictive of financial stress $[13,20]$. This study finds similar results: self-reported health status, insurance, and highest educational attainment were consistently predictive of financial stress. Those with poor/fair self-reported health, those with lower educational attainment, and those without insurance were more likely to experience financial stress. In addition, it was found that income level was determined to be highly predictive within skin cancer patients. Efforts to improve disparities should focus on these core determinants of financial stress, without ignoring the complex interplay of additional socioeconomic and structural factors.

Other studies have discussed the improvement and contextualization of care to those patients affected by lower socioeconomic factors and other social determinants. One option which has been discussed is routine screening for financial vulnerability across entire patient populations, with the goal of referral to community-based support and social assistance programs [6, 7, 21]. However, such interventions must be contextualized within existing administrative structures and the potential complexities of implementation, such as the growth of overhead costs or potential reinforcement of harmful stereotypes [7, 21, 22]. Some institutions have begun validating healthcare-related financial stress as a discrete patient-reported outcome metric, expanding opportunities for payment model innovation and more specific risk stratification [23, 24]. Other researchers have suggested teledermatology as a potentially useful tool to help the socioeconomically disadvantaged [8]. However, as the surgical nature of diagnosis and treatment of skin cancer requires in-person visits, teledermatology alone is inadequate to treat these patients. Additionally, technological solutions may be incomplete wherever the cost reductions are not effectively translated to financially struggling patients [2].

Our study has limitations. There were low percentages of minority patients within the sample of skin cancer patients answering the financial stress survey questions. While this smaller sample is reflective of previous research demonstrating a lower skin cancer prevalence in minority populations, adequate sampling is necessary to achieve statistical power to completely analyze financial stress within these groups [25]. Future studies with focused recruitment of minority skin cancer patients should be conducted to understand how financial stress may affect care and disparities within these populations. In addition, this study does not include financial stress information collected during the coronavirus pandemic and economic lockdown. These events have the potential to greatly affect the financial stress of the United States population and worsen disparities due to the pandemic's disproportionate effects on poorer and more diverse populations [26]. Finally, the nature of the cross-sectional study design limits the ability to draw a direct causal relationship between patient-reported financial stress and terminal outcome measures. Nevertheless, the present study is provided strength by a large sample size, nationally representative cohort, as well as the methodological rigor and sampling design of the NHIS dataset.

The present retrospective cross-sectional review of the 2013 to 2018 cycles of the National Health Interview Survey investigates the prevalence of financial stress among the skin cancer population within the United States. Where possible, additional resources directed towards at-risk skin cancer patients may best serve their unique socioeconomic contexts. Although financial stress has been hypothesized to correlate with poorer treatment outcomes within this patient population, the mechanism and magnitude of this relationship have yet to be rigorously studied. The degree to which skin cancer patients experience financial stress as a direct result of out-of-pocket dermatologic expenses also remains unclear. Whereas the present study is limited to a retrospective cross-sectional design, future investigations should organize a prospective multi-institutional cohort aimed at comparing the effects of various levels of financial stress on intermediate and terminal outcomes such as loss to follow-up, treatment failure, and survival. Additionally, future research efforts should analyze the effectiveness of the implementation of various financial assistance programs, 
along with patient-centered qualitative research to identify areas to more completely support patients.

\section{Conclusion}

A considerable proportion of skin cancer patients experience financial stress related to both healthcare and non-healthcare factors. This stress may limit treatment efficacy by forcing the postponement of care and decreasing the ability to follow dermatologist recommendations. These findings highlight the importance of contextualizing care of patients at risk of high financial stress to optimize patient experience and outcomes.

Author contributions All authors contributed to the study's conception and design. Material preparation, data collection, and analysis were performed by TBC. The first draft of the manuscript was written by TBC and TKJ, and all authors commented on previous versions of the manuscript. TBC takes responsibility for the paper as a whole.

Funding There was no specific funding for this research.

Availability of data and material The data are publicly available from the website of the National Center for Health Statistics (NCHS).

Code availability Upon request.

\section{Declarations}

Conflict of interest The authors declare no relevant conflicts of interest.

Ethics approval This study was deemed exempt by the University Hospitals Cleveland Medical Center Institutional Review Board.

Consent to participate Not applicable (does not involve human subjects).

Consent for publication Not applicable.

\section{References}

1. Zafar SY, Peppercorn JM, Schrag D et al (2013) The financial toxicity of cancer treatment: a pilot study assessing out-of-pocket expenses and the insured cancer patient's experience. Oncologist 18:381-390. https://doi.org/10.1634/theoncologist.2012-0279

2. Rothstein BE, Gonzalez J, Cunningham K et al (2017) Direct and indirect patient costs of dermatology clinicvisits and their impact on access to care and provider preference. Cutis 100:405-410

3. Chino F, Peppercorn JM, Rushing C et al (2017) Out-of-pocket costs, financial distress, and underinsurance in cancer care. JAMA Oncol 3:1582-1584

4. Apalla Z, Lallas A, Sotiriou E et al (2017) Epidemiological trends in skin cancer. Dermatol Pract Concept 7:1. https://doi.org/10. 5826/dpc.0702a01

5. Gilligan AM, Alberts DS, Roe DJ, Skrepnek GH (2018) Death or debt? National estimates of financial toxicity in persons with newly-diagnosed cancer. Am J Med 131:1187-1199.e5. https:// doi.org/10.1016/j.amjmed.2018.05.020

6. Fraze TK, Brewster AL, Lewis VA et al (2019) Prevalence of screening for food insecurity, housing instability, utility needs, transportation needs, and interpersonal violence by US Physician practices and hospitals. JAMA Netw Open 2:e1911514. https://doi.org/10.1001/jamanetworkopen.2019.11514

7. Davidson KW, McGinn T (2019) Screening for social determinants of health: the known and unknown. JAMA J Am Med Assoc 322:1037-1038

8. Cortez JL, Vasquez J, Wei ML (2020) The impact of demographics, socioeconomics, and healthcare access on melanoma outcomes. J Am Acad Dermatol. https://doi.org/10.1016/j.jaad. 2020.07.125

9. Qian Y, Johannet P, Sawyers A et al (2020) The ongoing racial disparities in melanoma: an analysis of the Surveillance, Epidemiology, and End Results database (SEER) database (19752016). J Am Acad Dermatol. https://doi.org/10.1016/j.jaad. 2020.08.097

10. Yang JJ, Maloney NJ, Cheng K, Bach DQ (2020) Financial burden in US melanoma patients from 1997-2015: racial disparities, trends, and predictors of high expenditures. J Am Acad Dermatol Patients. https://doi.org/10.1016/j.jaad.2020.07.051

11. National Center for Health Statistics. https://www.cdc.gov/nchs/ nhis/index.htm. Accessed 24 Sept 2020

12. Desai A, Jella TK, Cwalina TB et al (2021) Demographic analysis of financial hardships faced by brain tumor survivors. World Neurosurg. https://doi.org/10.1016/J.WNEU.2021.10.124

13. Mszar R, Grandhi GR, Valero-Elizondo J et al (2020) Cumulative burden of financial hardship from medical bills across the spectrum of diabetes mellitus and atherosclerotic cardiovascular disease among non-elderly adults in the United States. J Am Heart Assoc 9:e015523. https://doi.org/10.1161/JAHA.119. 015523

14. Jella TK, Cwalina TB, Sachdev R et al (2021) Prevalence, trends, and demographic characteristics associated with self-reported financial stress among head and neck cancer patients in the United States of America. Am J Otolaryngol 42:103154. https://doi.org/ 10.1016/J.AMJOTO.2021.103154

15. Hom GL, Cwalina TB, Jella TK, Singh RP (2021) Assessing financial insecurity among common eye conditions: a 2016-2017 national health survey study. Eye 2021:1-8. https://doi.org/10. 1038/s41433-021-01745-1

16. Cwalina TB, Jella TK, Zheng DX et al (2021) Utilization of health information technology among skin cancer patients: a cross-sectional study of the National Health Interview Survey from 2011 to 2018. J Am Acad Dermatol. https://doi.org/10.1016/J.JAAD. 2021.03.033

17. Butler SS, Winkfield KM, Ahn C et al (2020) Racial disparities in patient-reported measures of physician cultural competency among cancer survivors in the United States. JAMA Oncol 6:152-154

18. Rao JNK, Scott AJ (1987) On simple adjustments to Chi-square tests with sample survey data. Ann Stat 15:385-397. https://doi. org/10.1214/aos/1176350273

19. Koch GG, Freeman DH, Freeman JL (1975) Strategies in the multivariate analysis of data from complex surveys. Int Stat Rev 43:59-78

20. Yabroff KR, Zhao J, Han X, Zheng Z (2019) Prevalence and correlates of medical financial hardship in the USA. J Gen Intern Med 34:1494-1502. https://doi.org/10.1007/s11606-019-05002-w

21. Garg A, Boynton-Jarrett R, Dworkin PH (2016) Avoiding the unintended consequences of screening for social determinants of health. JAMA J Am Med Assoc 316:813-814 
22. Taylor LA, Tan AX, Coyle CE et al (2016) Leveraging the social determinants of health: what works? PLoS ONE 11:e0160217. https://doi.org/10.1371/journal.pone.0160217

23. de Souza JA, Yap BJ, Wroblewski K et al (2017) Measuring financial toxicity as a clinically relevant patient-reported outcome: The validation of the COmprehensive Score for financial Toxicity (COST). Cancer 123:476-484. https://doi.org/10.1002/cncr.30369

24. Jagsi R, Ward KC, Abrahamse PH et al (2018) Unmet need for clinician engagement regarding financial toxicity after diagnosis of breast cancer. Cancer 124:3668-3676. https://doi.org/10.1002/ cncr.31532
25. Gupta AK, Bharadwaj M, Mehrotra R (2016) Skin cancer concerns in people of color: risk factors and prevention. Asian Pac $\mathrm{J}$ Cancer Prev 17:5257. https://doi.org/10.22034/APJCP.2016.17. 12.5257

26. Adhikari S, Pantaleo NP, Feldman JM et al (2020) Assessment of community-level disparities in coronavirus disease 2019 (COVID19) Infections and deaths in large US metropolitan areas. JAMA Netw open 3:e2016938. https://doi.org/10.1001/jamanetwor kopen.2020.16938

Publisher's Note Springer Nature remains neutral with regard to jurisdictional claims in published maps and institutional affiliations. 